University of Nebraska - Lincoln

DigitalCommons@University of Nebraska - Lincoln

February 2002

\title{
Improvement in Quantity and Quality of Prevention Measurement of Toddler Injuries and Parental Interventions
}

\author{
Lizette Peterson \\ University of Missouri \\ David DiLillo \\ University of Nebraska-Lincoln, ddilillo@unl.edu \\ Terri Lewis \\ University of Missouri \\ Kenneth Sher \\ University of Missouri, Sherk@missouri.edu
}

Follow this and additional works at: https://digitalcommons.unl.edu/psychfacpub

Part of the Psychiatry and Psychology Commons

\footnotetext{
Peterson, Lizette; DiLillo, David; Lewis, Terri; and Sher, Kenneth, "Improvement in Quantity and Quality of Prevention Measurement of Toddler Injuries and Parental Interventions" (2002). Faculty Publications, Department of Psychology. 154.

https://digitalcommons.unl.edu/psychfacpub/154
}

This Article is brought to you for free and open access by the Psychology, Department of at DigitalCommons@University of Nebraska - Lincoln. It has been accepted for inclusion in Faculty Publications, Department of Psychology by an authorized administrator of DigitalCommons@University of Nebraska - Lincoln. 


\title{
Improvement in Quantity and Quality of Prevention Measurement of Toddler Injuries and Parental Interventions
}

\author{
Lizette Peterson \\ David DiLillo \\ Terri Lewis \\ Kenneth Sher
}

University of Missouri

\begin{abstract}
Injury is the leading killer of children in the United States, yet little research has focused on this vital subject. One of the distinct barriers to injury prevention is the absence of effective assessment devices. Epidemiological aspects of injury have been assessed, but these provide little information at a level sufficient to allow conclusions about potential behavioral prevention methods. This paper describes an alternative, the Participant Event Monitoring (PEM) system. In this paper, the PEM system is used to examine a sample of 170 toddlers (ages 18 to 36 months), over a 6-month period, resulting in data on over 4,200 injuries, 1,000 proactive interventions, and 300 reactive interventions. PEM involves a structured interview, producing detailed information concerning measures of antecedents, events, and consequences of injury. Sample PEM data are included. Its ultimate goal is to guide effective interventions to decrease childhood injury.
\end{abstract}

Injuries are the leading killer of children in the United States, taking more lives than the next nine leading threats to children's health (Rivara, 1982). There are 14 million children injured every year (National Institute for Health Care Man-

We first need to cite our funding agency, NICHD Grant \#5R01HD25414-07. Connie Popkey assisted in multiple drafts of the paper and over 30 undergraduates assisted in coding the data. They deserve our sincere thanks. The interviewers Bonnie Benson, Jennifer Collins, Amy Damashek, Julie Long, Shannon Stanton, Lisa Wischmeyer, and Wendi Marien, M.S., all did consistently superb work as interviewers. The University of Missouri Pediatrics Department assisted us in recruiting, as did day care centers and parenting groups (such as "Parents as Teachers"). Jeff Crowson assisted in initial data entry and cleaning. Finally, our greatest thanks go to the women who spent such time and effort as participant observers. Without their training and willingness to accurately report on their children's behavior, such research would not be possible. 
agement, 2000) and 600,000 of these children are hospitalized, 30,000 are permanently disabled, and 22,000 die from injuries (Rodriguez, 1990). Costs for treating unintentional childhood injury are estimated at over $\$ 14$ billion in medical costs, $\$ 1$ billion for other resources, and $\$ 66$ billion in present and future work losses (Miller, Romano, \& Spicer, 2000). Yet, prevention of childhood injuries has rarely been the subject of health care research. The majority of past work has been conducted by public health experts and epidemiologists, and has linked such factors as geography, ethnicity, and socioeconomic status to injury (Baker, O'Neill, Ginsburg, \& Li, 1992). Although providing a necessary description of the broad demographic risk factors associated with injury, such research has rarely yielded the specificity of data needed to understand the mechanisms of injury that could guide effective, family-level, behavioral methods of reducing childhood injury.

One way of conceptualizing the prevention of childhood injuries is to place them on a continuum beginning with societal-level interventions involving no caregiver effort to interventions relying solely on caregiver effort. To understand this continuum, it is helpful to consider examples at each of five levels:

- legislative changes (e.g., mandating that manufacturers produce only cribs with slats sufficiently close together that an infant's head could not fit in between them; Consumer Product Safety Commission, 1979);

- product changes with minimal caregiver effort (e.g., encouraging parents to set water heaters one time to $125^{\circ} \mathrm{F}$ to insure prevention of scalding bums to children);

- environmental changes with some degree of repeated effort by caregivers (e.g., installing a child gate that must be closed each time it is used but requires only that and no subsequent supervision);

- learning safe behavior involving much caregiver or child effort and supervision (e.g., assisting parents in the proper use of child safety seats in cars and in their current use each time the child rides in the car); and finally,

- efforts involving maximum caregiver effort (e.g., prevention of child drowning in a bathtub or pool by continuous supervision; never leaving the child even for a single minute near water).

The lower levels on the continuum are universally known to be the most effective, as no additional caregiver efforts are required, only the alteration of product safety. However, such interventions may be perceived by some as unnecessarily limiting individual freedom. Lobbying by powerful political groups has undermined the utilization of many effective barrier interventions (e.g., trigger locks on hand guns, the banning of mobile baby walkers; Peterson \& Roberts, 1992). Individual freedom in the absence of government regulation is one of our country's most cherished convictions, but for child safety this means the caregiver assumes total responsibility for children's safety; most methods of maintaining child safety, such as continuous supervision, are so difficult to conduct in terms of cost of con- sistent parental effort (Canadian Children's Safety Network, 1996) that they are unlikely to be used with peak efficiency. Thus, there exists a constant tension between caregivers maintaining their complete right to make child-care decisions and the right of the community to protect its children by mandating caregiver behavior. Such decisions have often been influenced by the costs of intervention, with communities being more willing to impose rules on the caregiver if the demands of the rules are low. The tension can be described in this fashion: On one end of the scale, injury prevention can be achieved effectively, but with some cost to personal freedom; at the other end of the scale, high levels of personal freedom are preserved, but currently, because of our lack of knowledge concerning ways of ensuring consistent caregiver effort, some degree of effectiveness in maintaining children's safety may be sacrificed. It is only recently that behavioral (Scheidt \& Workshop Participants, 1988), more active (Shields, 1997) methods have received increased attention as methods of increasing effectiveness and concurrently decreasing effort or costs. This article describes a method of considering all levels of prevention, but unlike most past articles on prevention, focuses especially on the high end (maximum parental effort but attempting to also ensure maximum effectiveness) of the scale.

Those few studies investigating spontaneous intervention efforts used by caregivers typically focus on situations in which parental action is the only way in which children's safety can be maintained. The few data that do exist suggest that parents who are aware they are being observed rely on relatively continuous supervision of ongoing child behavior and they focus on children's risk behaviors as a more frequent target for intervention than other behaviors (e.g., more on safety than on social behaviors such as "please," or self-care such as brushing teeth). There may also be a shift toward decreasing such interventions from younger (1 year) to older ( 2 to 3 years of age) children. For example, Gralinski and Kopp (1993) asked mothers of toddlers to complete behavior checklists identifying child behaviors that elicited their typical parental interventions. They reported that 2 to 4 times more behavioral interventions with children concerned safety rules in comparison with other elicitors of parental attention, but the number of safety interventions unexpectedly decreased in children from 13 to 30 months of age. Similarly, Power and Chopieski ( 1986) observed more maternal restrictions placed on child injury behaviors than other behavior problems, with an average of 4.9 restrictions in each 45-minute session.

Studies like these suggest that, left to their own devices, parents tend to rely on brief behavioral interventions to keep children safe from physical harm. In describing their interventions they anecdotally often seem relatively unskilled. Past research has shown that behavioral methods focusing on teaching a single preventive skill and using a strong consequence can be very effective (Peterson, Mori, Selby, \& Rosen, 1988). There are, however, few studies currently in the literature demonstrating a widespread approach to prevention that has been effective. 
Behavioral changes that would involve altering general parenting strategies such as supervision, distraction from hazards, etc., at least in theory, can be very effective (Finney et al., 1993) and can also be broad, in terms of application to different types of injuries that can be prevented. Why have such broad tactics failed to be the subject of research? Many researchers have suggested that the absence of a reliable and sensitive measurement methodology that might reveal the situations in which changes in parenting are required is a major deficiency in the area (Deal, Gomby, Zippiroli, \& Behrman, 2000); this deficiency may limit the possibility to effectively study broad-scale behavioral prevention. Perhaps if there were a more comprehensive system of assessing the situations in which injuries take place, the task of matching injury risk with type of preventive intervention would be more straightforward. Prior methods, however, have failed to produce the behavioral specificity of information necessary to understand the complex behavioral mechanisms thought to underlie childhood injuries, mechanisms that represent some of the most promising points of intervention for future preventive efforts. The present paper describes a methodology focused on achieving this goal. By having caregivers record in detail the primary behavioral and environmental events that precede, coincide with, and follow the injury event, heretofore unknown factors contributing to injury risk may be identified.

\section{Current Sources of Data Examining Etiology of Injury}

\section{Random Community Samples}

Some data relevant to childhood injury occurrence that may influence caregivers' decisions about appropriate behavior underlying injury have been obtained by simply selecting a random community sample. The data collected from these samples may reveal what drives the types of preventive interventions conducted by caregivers. For example, beliefs about the extent to which injuries are a threat to children may influence all levels of the continuum, from altering the environment to increasing supervision. For example, Eichelberger, Gotschall, Feely, Harstad, and Bowman (1990), in a national sample, and Peterson, Farmer, and Kashani (1990), in a smaller community-based sample, found that parents were concerned with a variety of child hazards. However, when asked about specific hazards, they listed concerns such as drug use and stranger abduction, which are legitimate concerns, but which harm far fewer children than the leading cause of death, physical injury. Such priorities are likely to result in parents who do not give injuries the resources and effective preventive efforts they deserve.

Parents may have inaccurate beliefs about the extent to which children are at risk for injury, both because of inaccurate beliefs stemming from sources such as the media, health care professionals, and friends, but also because they do not accurately recall the number of injuries their own child has sustained, if the injuries were not serious. Parents seem to recall that a medically attended injury occurred, but they do not recall the incident in sufficient detail, and this makes it difficult for them to plan to prevent future injuries. Further, in community samples parents do not seem able to readily recall even the occurrence of nonmedically attended injuries, let alone the details surrounding the injury (Peterson, Moreno, \& HarbeckWeber, 1993). The use of large-scale community samples remains rare, partially because of their potential for inaccuracy (individuals feeling a need to report more safety habits than they actually practice or having a poor memory for relevant events) and the expense of such data. Because the present method produces high accuracy concerning the occurrence of all events (and thus yields records of a large number of events), a very large number of participants is unnecessary to study a sizeable sample of injuries.

\section{Medical Records}

Children's medical records are perhaps the most frequently used source of epidemiological data regarding children's injury. However, like data from community samples, data from medical records are likely to have varying levels of accuracy and too often lack details involving the antecedents and consequences of injury (Christoffel et al., 1992; U.S. Advisory Board on Child Abuse and Neglect, 1995). Further, the definition of an injury that requires medical attention is entirely subjective, unless sufficiently serious to result in ratings typical to emergency room triage systems, where the lowest items on the scale are broken bones or torn ligaments, tissue damage clearly documented by X rays or other medical tools. A caregiver may bring a child into a medical setting for treatment of even a minor injury because of the parent's or the child's idiosyncratic behavior (e.g., the parent's oversensitivity to blood, the child's crying readily, etc.). This can result in the inclusion of injuries with very little or no tissue damage or the exclusion of injuries with significant tissue damage, and the errors that parents make in regarding such injuries as similar to minor injuries.

In some cases, this seemingly random error can be very systematic and can become a serious problem if the artifact introduced is associated with a risk factor for the injury. For example, parents of low socioeconomic status (SES) are likely to live in environments that are high in terms of number of safety hazards (lead, traffic, unsafe methods of heating), and offer less in terms of environmental and behavioral methods of injury prevention (e.g., fewer safety latches and smoke alarms, higher proportion of number of children to caregivers; Peterson \& Brown, 1994). Yet, low SES individuals are also less likely to seek medical care because of fewer consolidated relationships with the medical community and greater difficulty in obtaining medical care. Thus, it is difficult to determine the true relationship of injury rate and SES if only medically attended injuries are considered.

SES is but one potentially confounding variable, one that is easy to distinguish in data collected from medical records. There are an unknown number of factors influencing subjective definition of injury severity, including issues such as (a) how informed a parent is concerning injury seriousness, (b) a child's physical and 
emotional reaction to the injury, and (c) the caregiver's idiosyncratic evaluation of physical factors such as swelling and bleeding. There may be still other factors, such as acceptance and affiliation with a medical caregiver, cost of transportation, and care of other children, that influence the decision to seek medical assistance. Behavioral methods of assessment can assist in identifying the variables specifically related to factors such as injury risk, injury severity, and the act of seeking medical assistance.

\section{Epidemiological Data on Injury Fatalities}

Some epidemiologists have attempted to solve the problem of a lack of objectivity in identification of cases by focusing solely on disabling or fatal injuries. In this way, the investigator is studying a well-defined type of tissue damage, one in which the definition is independent of subjective child or caregiver factors. However, limiting study to injuries with serious outcomes ignores the larger number of injuries that resulted in less tissue damage, yet may have been debilitating, stressful, or had the potential for serious injury. Many minor injuries could have been far more serious and could yield data regarding a range of potentially serious injuries. Further, even minor injuries involve factors such as child pain, parental anxiety, and often blame. This can result in anxiety, dysphoria, or marital conflict as different individuals consider why the child is upset, hurt, bleeding, etc., and how this could have been even worse. Thus, minor injuries are worthy of study in their own right.

\section{New Behavioral Alternatives}

As has been noted, injuries are fairly ubiquitous events in the lives of children. Yet, because they are sufficiently low base-rate events, it is unreasonable to utilize conventional observational methods. Similarly, beeper or other systems used for more frequent events such as smoking urges or depressive symptomatology seem impractical due to the unpredictability of injury and the absence of behavioral patterns characteristic of some other low base-rate occurrences. It seems unlikely that a parent could take sufficient time to provide an extensive interview on the experience at the time of the injury. A promising alternative method of assessing the correlates of childhood injury is to teach caregivers how to observe and report the antecedents, injuries, and consequences of injury as they occur.

To this end, we have derived Participant Event Monitoring (PEM), which utilizes parental monitoring and recording of injury events directly after they occur (see detailed description in Peterson, Brown, Bartelstone, \& Kern, 1996). A structured and standardized set of questions is presented to the caregivers within 2 weeks by skilled clinical interviewers. Caregiver records and the structured interviews allow for the collection of a large amount of relatively specific data for all levels of severity, without the subjectivity present in other methods of injury assessment
In the initial research examining this methodology, 8-year-old children and their mothers participated in a year-long study (Peterson, Bartelstone, Kern, \& Gillies, 1995). Children of this age were selected because they could be questioned about injuries that occurred in the caregiver's absence without risking the requirement of reporting caregiver neglect, as 8-year-olds can legally be left unsupervised in this state. These children also were capable of acting as their own independent participant observers, allowing us to correlate child data with the mothers' data to establish preliminary reliability of the injury reports. The child's report of type, severity, and treatment of a given injury allowed us to index reliability by comparing child reports to mother's data, and these indicators suggested reliability was high. Because of the need to establish reliability, the preliminary study examined school-aged children and thus was an advantage. However, younger children are at much higher risk because they are just learning to walk and talk. Children of this age do not have the cognitive maturation to have internalized rules. Thus, it is unreasonable to expect them to conform to injury prevention rules and regulations in the caregiver's absence. However, parents often expect that they will (Peterson, Gable, Doyle, \& Ewigman, 1997). For these reasons, not only are toddlers the population at greatest risk for injury (Baker et al., 1992), but they present special problems for skilled parenting and the present report focused on toddlers and preschoolers. Also, the earlier study lacked many of the advantages of the present study, which will be described later. These include a case control design to compare injury antecedents and consequences with similar settings without injury, and data on several types of injury prevention interventions. Thus, in many ways, this study breaks new ground in the injury prevention field.

The purpose of the present study is to provide a broad-based demonstration and extension of aspects of toddler and preschooler injuries that can only be obtained with a method such as PEM. As the following data demonstrate, this method allows researchers to focus on issues such as number of injuries and all levels of injury severity, in addition to control conditions and interventions not evaluated before. The occurrence of all injuries may be evaluated within mutual relationships of child gender, age, injury severity, potential severity of injury, and the relationship of the extent to which the caregiver sought medical attention to the other attributes of the injury. Finally, researchers can explore caregiver responses to injuries, both reactive actions (those that occur following an injury to prevent reoccurrence) and proactive actions (those that happen before an injury to prevent any occurrence).

\section{Method}

\section{Participants}

Mothers of children ages 15 to 18 months and 33 to 36 months were recruited from the general community using a variety of strategies, including flyers distributed to day care centers and parent groups (e.g., "Parents as Teachers"), a patient telephone list from a local pediatric center serving primarily low-income fami- 
lies, and weekly advertising both in the newspaper and local giveaway ad sheets. Mothers rather than fathers were recruited, as they were more likely to be the primary caregiver. Mothers were contacted and informed about the demands of the project, as well as reimbursement for their effort (\$21 per week for 26 weeks plus \$25 for a final laboratory session).

The mothers were then screened for eligibility. In addition to child age, mothers were required to be the primary caregiver, fluent in English, have no other children within 10 years at the time of recruitment (a toddler, with a 12-year-old sibling, for example, was still regarded as a singleton) and not planning to leave the area for more than 2 weeks during the study. Children between 15 and 18 months of age and between 33 and 36 months of age were selected because previous research (e.g., Gralinski \& Kopp, 1993) indicates that these age ranges are most likely to receive differential parental injury prevention. Children had no major disabilities, no overnight hospitalizations for past injury, and no known history of child maltreatment. The final 170 target children included approximately equal numbers of boys $(54 \%, n=92)$ and girls $(46 \%, n=78)$. Mothers were predominantly Caucasian (91\%), married (83\%), in their mid-to-late 20s $(M=28.8$ $S D=4.43$ ), well-educated (nearly half, $47 \%$, had graduated from college), and employed 30 or more hours per week $(42.4 \%)$. These were middle-class families (modal family income was over $\$ 55,000$; the lowest point of the range less than $\$ 15,000$ to the highest point of the range over $\$ 55,000$ ). This population was very similar to the population serving in the initial test of the PEM (Peterson, Brown, et al., 1996). Despite a concerted effort to recruit low-income families, many potential participants could not be reached, would not complete the eligibility screening, or did not meet eligibility requirements. ${ }^{1}$ However, the mothers in this study may represent the population of women who are most likely to contact their primary-care physician in the event of an injury and thus allowed us a more sensitive test of the extent to which actual tissue damage is the primary cause of making medical contact.

\section{Interviewers and Coders}

Six postbaccalaureate female research assistants were extensively trained in using the script for the PEM method (Peterson, Brown, et al., 1996). These women interviewed approximately 14 mothers every other week and one postmaster's student interviewed 1 woman every other week. The same individual interviewed the same mother each week, with exceptions due to travel or illness, in which case interviewers would substitute for one another. The second author monitored ini-

${ }^{1}$ Many of the women for whom we had telephone numbers from the pediatrics clinic or from messages from their own calls to our laboratory could not be contacted $(n=362)$ and some were unwilling to continue the interviews long enough to determine eligibility $(n=61)$. Of those screened, 307 were ineligible, and of those eligible participants $(n=275), 181$ started actual participation. Once enrolled. the large majority $(n=161,89 \%)$ finished all 6 months, 9 finished at least 6 weeks (amounting to the first three interviews), and 11 dropped out before the third interview. tial interviews through audiotapes. Over 30 male and female undergraduate students, uninformed concerning study hypotheses and the injury literature, earned course credit by applying empirically derived codes to the interview data that required categorization.

\section{Training of Injury Observation}

Mothers were carefully trained in the parameters of the injuries they were to observe. An injury was defined as any specific outcome of an event that involved tissue damage that could be visually or tactilely ascertained such as bumps, bruises, skinned knees, cuts, etc., that lasted at least 24 hours. Regardless of the mother's subjective concern about the child's reaction, such as crying, an event was not regarded as an injury unless at least minor tissue damage could be observed.

Parents completed monitoring sheets as personal reminders each time an injury occurred, with a small number of details required to recall the event. Our intent was to limit the amount of effort, but have sufficient information to cue detailed recollection during the biweekly clinical interview. The mothers recorded aspects about the injury such as date, time, type of injury and the location of the injury, which was indicated by drawing an $\mathrm{X}$ on the form of a child provided on their monitoring sheets. Mothers were also asked to indicate the actual size of the injury on a separate sheet of paper by drawing it so it could be measured in millimeters. The mothers recorded the antecedents, events, and consequences of the event, including information such as the caregiver's proximity to the child at the time of the injury and the caregiver's own feelings about the injury.

\section{Interview Structure}

A standardized 2-hour interview was held every other week for 6 months. The interviews always lasted 2 hours in order to discourage mothers from failing to report injuries and interventions in an effort to limit interview time. When there were insufficient injuries and interventions to fill the entire 2-hour time frame, mothers completed a variety of questionnaires assessing maternal, child, and family characteristics.

Injuries. When interviewing about injury events, our past work suggests that fewer injuries are reported when an open-ended question (i.e., "Did your child sustain any injuries?") rather than a specific question (e.g., "Did your child sustain any bumps or bruises, any cuts or scrapes?") is used. Therefore, we asked questions regarding 18 specific injury types (see Table I). If the mother indicated that an injury of that type had occurred, a list of probes followed, including date, time, and location of injury, detailed description of the tissue damage involved, and the antecedents and consequences of the injury, including the child's emotions and any treatment received. The location of the caregiver and the caregiver's attributions of fault were also queried. Some of these data were Likert ratings directly from the parent (emotions of the child), and others were slightly more complex 
TABLE 1

INJURY CATEGORIES SCORED BY THE MINOR INJURY SEVERITY SCALE

\begin{tabular}{lrr}
\hline Injury Category & & $\begin{array}{c}\text { Frequency } \\
\text { (Percent of } \\
\text { Total }\end{array}$ \\
\hline Bruise/bumpes)
\end{tabular}

Note. $N=3,848$. Categories were not scored for 442 injuries. Table does not include injury categories with a zero frequency. Injury categories with a zero frequency include Firearm/Bow and Testicular impact.

and were rated by the interviewer on site. A third type was quite complex, requiring training in the different parameters of each question, and these were rated by coding teams. Table 2 contains examples of those kinds of ratings obtained from the injury probes. Given the large number and range, they will not be detailed here, but can be obtained from the first author.

Interventions. Parents were asked about any interventions they completed that were either reactive or proactive. Previous literature (Gralinski \& Kopp, 1993; Power \& Chopieski, 1986) has shown that parents may interact with their children several times in an hour with brief interventions to prevent injuries (e.g., barriers such as locking an item up, maternal behavior change such as moving a poison from beneath the sink to a high shelf, and teaching such as "No, don't touch that, it is hot"), and thus it was not possible to measure so many interventions over the twelve 2-week periods effectively. We therefore focused only on interventions lasting over 30 seconds (showing clear parental effort) within the types of interventions (barriers, parent behavior, teaching child behavior) used in past research The date of the intervention and a detailed description of each intervention was obtained; if the mother reported an intervention, the date, time, and type of the injury that cued the current intervention was obtained. For both types of interven-
TABLE 2

EXAMPLES OF INTERVIEW PROBES

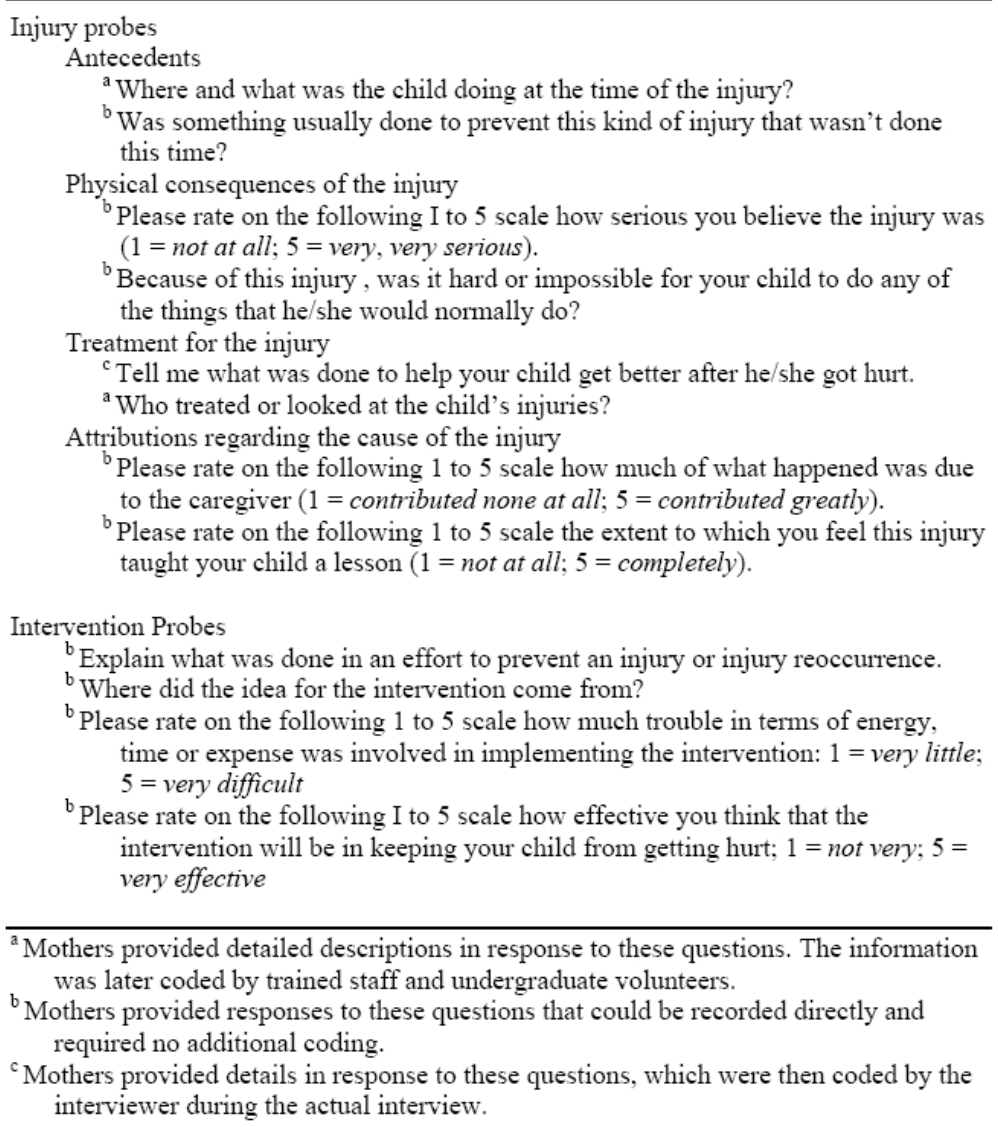

Physical consequences of the injury

${ }^{b}$ Please rate on the following I to 5 scale how serious you believe the injury was $(1=$ not at all $; 5=$ very, very serious $)$.

${ }^{b}$ Because of this injury, was it hard or impossible for your child to do any of the things that he/she would normally do?

Treatment for the injury

${ }^{\mathrm{c}}$ Tell me what was done to help your child get better after he/she got hurt ${ }^{a}$ Who treated or looked at the child's injuries?

Attributions regarding the cause of the injury

${ }^{6}$ Please rate on the following 1 to 5 scale how much of what happened was due to the caregiver $(1=$ contributed none at all $; 5=$ contributed greatly $)$.

${ }^{b}$ Please rate on the following 1 to 5 scale the extent to which you feel this injury taught your child a lesson $(1=$ not at all $; 5=$ completely $)$.

Intervention Probes

${ }^{b}$ Explain what was done in an effort to prevent an injury or injury reoccurrence.

${ }^{\mathrm{b}}$ Where did the idea for the intervention come from?

${ }^{b}$ Please rate on the following 1 to 5 scale how much trouble in terms of energy, time or expense was involved in implementing the intervention: $1=$ very little; $5=$ very difficult

${ }^{\mathrm{b}}$ Please rate on the following I to 5 scale how effective you think that the intervention will be in keeping your child from getting hurt; $1=$ not very; $5=$ very effective

\footnotetext{
${ }^{a}$ Mothers provided detailed descriptions in response to these questions. The information was later coded by trained staff and undergraduate volunteers.

${ }^{b}$ Mothers provided responses to these questions that could be recorded directly and required no additional coding.

${ }^{c}$ Mothers provided details in response to these questions, which were then coded by the interviewer during the actual interview.
}

tions, the origin of the idea for the intervention was described, the rating of the estimated highest cost (in time, effort, and money) for the intervention was obtained, and finally, the most effective maternal rating, if more than one intervention, was conducted. See Table 3 for some examples of origins mothers listed for proactive interventions; as can be seen, most came from the mother herself, either thinking of the intervention by herself (listing it as common sense or just occurring) or concern about a near injury, which indicates a quarter or more of these interventions classified as proactive seem to stem from past research and may be better thought of as reactive.

Control condition. A case-crossover (Maclure, 1991) component was included as part of the PEM method to determine the relative risk of injury for a number 


\section{TABLE 3}

Maternal RePorts ReGarding the SoURCE of IdEAS For PROACTIVE INTERVENTIONS

Source

Common sense/observation (e.g., taking a knife away from a 2-year-old) Own child almost got hurt

Parent (mother, father, mother-in-law, father-in-law) $324(26.0)$

Popular press media (newspaper/magazine/book)

Friend/relative

Observational learning

Television

Partner/husband

Physical or health care professional

A different child was injured (e.g., child in the neighborhood)

Health Department pamphlet or other government printing

Neighbor

Own child got hurt prior to this study and did not want child to get hurt again

Radio

Note, $N=1,246$

of circumstances, including supervision level, household location, and child activity, to name a few. Circumstances surrounding no-injury situations were obtained by having mothers record data, identical to those obtained under injury occurrences, at a prearranged time and date that corresponded to the most severe injury reported in the previous interview. Because participants serve as their own controls in such a design, sampling biases and confounds due to subject characteristics are minimized (Louis, Lavori, Bailar, \& Polansky, 1984). The inclusion of this component is an important extension of the original PEM method and will allow us to compare injury and no-injury situations in order to identify factors or situations that are unique to injury situations.

Assessment of potential artifacts. It is possible that some forms of data, actually unrelated to the current data on children's injuries could influence mothers reports of number, severity, consequences, etc., of injuries. For example, changes in the child's health, new safety ideas that had been obtained, any peers known to have been hurt since the last interview, increases in concerns regarding childhood injury as a result of participating in an injury study, and the number of minor (less than 30 seconds) interventions the parent estimated may have influenced the number of injuries or interventions reported. Measuring these allows us to assess and to remove, if necessary, such confounds should they be shown to be related to other reports of injuries or interventions.
Anonymous feedback. Several months after participating in the study, women returned to the laboratory and completed several additional measures. One of these, most relevant to the present study, was the Project Feedback Form. This 42item questionnaire was guaranteed to be anonymous and could not be linked to participants, either by code numbers or names. This asked participants how well they had followed instructions, whether they had ever falsified information either by deleting or adding injury or intervention events, and whether they had completed data forms appropriately (at the time of the injury or directly before the interview, etc.). It provided an index of the extent to which the data were a valid indication of parents' understanding and recollection of events.

\section{Specific Measures}

Minor Injury Severity Scale (MISS; Peterson, Heiblum, \& Saldana, 1996; Peterson, Saldana, \& Heiblum, 1996). The MISS is an objective, reliable measure regarding the actual tissue damage incurred on a 0 (no tissue damage lasting 24 hours) to 6 (a disabling injury or death) scale, specific to the 18 types of injury shown in Table 1. Raters used information such as size, shape, depth, blood loss, and location on the body to create a score. Independent raters reliably applied the scale. First, type of injury was rated (kappas for pairs of raters ranged from .68 to .98 ), then injury of severity was rated (interclass correlations for pairs of raters ranged from. 79 to .92 )

Potential Severity Index (PSI). The PSI was created to allow researchers to evaluate the circumstances surrounding an injury and provide a global rating of how potentially serious an injury might have been (e.g., Peterson, Brown, et al., 1996). Trained coders used physical parameters such as height, hardness or sharpness of surfaces, speed, part of body contacted, etc., to yield a I (the injury never could have been very serious) to 5 (the injury might have been disabling or fatal) to make these determinations. Injuries were coded with acceptable levels of accuracy (interclass correlations for pairs of raters ranged from .61 to .82).

These two measures will be used to demonstrate how the PEM might be employed to understand the extent to which actual injury is related to potential injury. However, it seems important to clarify the breadth of the system by briefly describing other variables measured.

Reliability information for other measures. In order to assess the reliability of mothers' reports, interviewers' judgments, and coder agreement, 44 randomly chosen families, at a randomly chosen interview, were interviewed a second time by someone other than the family's primary interviewer. Of the 44 interviews, 37 included one or more injuries. Ninety-three injuries were reported by both raters, 60 of which were probed in detail. Reliability was not calculated for items where the number of cases was fewer than 20, where the response was a constant, or where one rater but not both endorsed a category. The following paragraphs detail reliability figures from these 44 reliability interviews by the reporter (e.g., mothers, interviewers, coders). 
For a number of items, mothers provided ratings or dichotomous responses for several of the interview questions (e.g., "Was the child's behavior typical or unusual?"). Reliability for continuous data was calculated by interclass correlations; categorical data were calculated by kappa. For the 39 items that were continuous in nature, the median ICC was $.81(M=.76$; range $=.07$ to .99$)$. For dichotomous or categorical questions, the median kappa was $.69(M=.66$; range $=.28$ to .85$)$.

Based on descriptions provided by the mother, interviewers provided ratings for several questions at the time of the interview (e.g., "How informed was Mom about the injury?"). Reliability was assessed for the primary and reliability rater. The median ICC for the 4 continuous items was $.74(M=.76$; range $=.63$ to .91$)$, and the single kappa was .80 .

Finally, both the primary and reliability interviewers wrote down descriptions of mothers' responses to several questions (e.g., "Where was the caregiver at the time of the injury?"). Undergraduate assistants and one B.S.-level staff person coded responses for the primary and reliability interviews. The majority of these items (with one exception) were categorical codes that required little training. For example, raters coded mothers' responses to the question, "Who was taking care of the child at the time of the injury?" using a list developed by the primary investigator. Responses from the primary interview and secondary interview were rated by different coders. The median kappa for the 5 items was $.73(M=.69$; range $=.45$ to .97$)$.

\section{Results}

\section{Descriptive Data}

Injuries. We obtained data representing a total of 4,290 injuries. The mean number of injuries per family per biweekly interview was $2.15(S D=1.29$; range $=0$ to 15 per interview). Of the 4,290 injuries, 2,485 were probed (meaning specific data related to the actual injury, the antecedents of the injury, and consequences of the injury were obtained). The remaining 1,805 injuries were not probed, either because the mother did not know how the injury occurred $(84 \%)$ or the interviewer was unable to probe the injury because the interview would have exceeded the 2-hour time constraint (16\%). Using the injury categories from the MISS, the majority of these injuries was bumps and bruises $(34.8 \%)$ or scrapes and scratches $(34.6 \%)$.

Because our previous experience suggested that children sustain more injuries in the summer months, we combined the initial date of participation into two groups, those beginning in the spring/summer months (April to October) and those beginning in the fall/winter months (November to March). In order to assess the effect of our dependent variables on injury rate, a 2 (child age) by 2 (child gender) by 2 (season of study enrollment) by 12 (interview) repeated-measures ANOVA was conducted for the 161 families who completed all 12 interviews. Probabili-

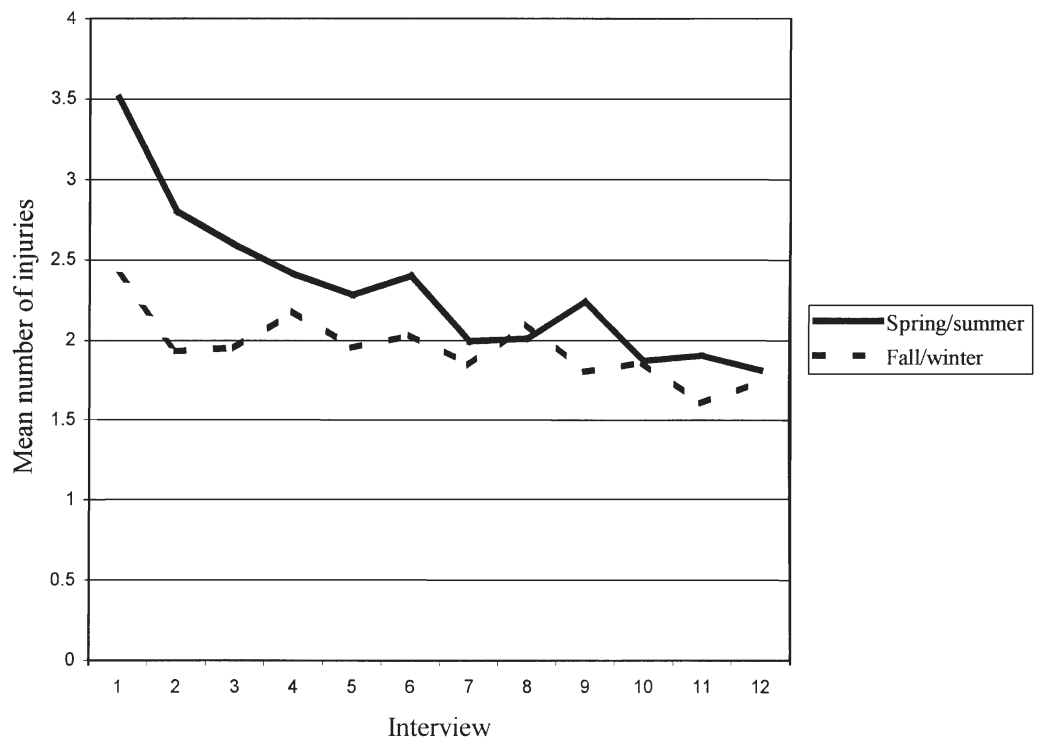

FIG. 1. Mean number of injuries per interview by season.

ty levels were adjusted using the Greenhouse-Geisser correction. ${ }^{2}$ Between-subject analyses indicated a main effect for child gender, $F(1,153)=4.16, p<.05$. As seen in most past research, boys consistently received more injuries on average per interview than did girls $(M=2.37, S D=1.36 ; M=1.94, S D=1.18$, respectively).

Within-subject analyses indicated that reports of injuries tended to decrease over time, $F(1,1683)=8.68, p<.05$; however, a significant Interview $\times$ Season interaction, $F(11,1683)=2.53, p<.05$, indicates that there may be a greater decline in injury rate for those families who began participation in the spring/summer months than those families who began in the fall/ winter months (see Figure 1). We found a similar shift across seasons in our previous research (Peterson, Brown, et al., 1996). The decrease in reports of injuries is not consistent with a normative decline associated with development (i.e., age) as we failed to detect an effect for child age, $F(1,153)=.17, p>.05$. Analyses also failed to reveal main effects for season or any of the 2- and 3-way interactions. The exact cause of the reduction in injury reports is unclear but may reflect a reactive reporting bias at-

${ }^{2}$ We applied the classical approach to repeated-measures ANOVA (with Geisser-Greenhouse correction for violations of sphericity) as opposed to the multivariate approach to repeated measures because it makes a number of contrasts (e.g., tests for polynomial trends) more tractable and makes it simpler for meta-analysts to infer specific effects of interest in secondary analyses. 
tributable to the interview demands or an increase in awareness of unsafe situations resulting in more injury prevention efforts.

Injury severity (MISS). The severity of injuries was coded using the MISS. As noted earlier, the MISS allows coders to objectively rate the severity of each injury as a function of tissue damage, blood loss, and location on the body. These ratings are independent of the course of action taken to treat the injury (e.g., doctor visit), child factors (e.g., amount of distress), or maternal factors (e.g., high arousal in response to blood). Although the MISS is a 7-point scale $(0=$ no damage to 6 = permanent disability or death), the range for this sample of injuries was 1 to 5. This was because, by definition, to be reported in the study, tissue damage had to be at least 1 and no injury in the present study was severe enough to be permanently disabling or fatal, which would receive a MISS score of 6 . Sufficient data were obtained on 3,848 in juries to allow coders to quantify the degree of severity of the injury. The mean MISS rating for this sample of injuries was 1.5 ( $S D=$ $.64)$, indicating that most of the injuries reported were fairly minor, with the majority receiving a score of $1(57.6 \%)$ or $2(35.4 \%)$ and relatively few injuries receiving a score of $3(6.6 \%), 4(.14 \%)$, or $5(.07 \%)$. Injuries receiving a score of 5 on the MISS $(n=3)$ included a fracture of the forearm, a dislocated elbow, and a skull fracture, all of which received medical attention.

Potential Severity Index (PSI). Trained coders assessed the situation surrounding the injury and coded the worst possible injury that could have resulted under the same conditions as the actual injury. Higher values indicate the injury had greater probability of resulting in death or permanent disability, while lower values indicate that even the worst injury outcome would not have resulted in death or disability. Sufficient data were obtained from mothers to allow coders to rate 2,483 of the injuries reported on this 1 to 5 scale. The mean PSI rating for these injuries was $2.57(S D=.87)$. The distribution of scores is as follows: $2.9 \%$ of injuries received a score of $1 ; 55.9 \%$ received a score of $2 ; 25.7 \%$ received a score of $3 ; 11.8 \%$ received a score of 4 ; and $3.7 \%$ received a score of 5 . It is particularly important to note that nearly $16 \%$ of the injuries had the potential to be very serious $(\mathrm{PSI}=4)$ or fatal $(\mathrm{PSI}=5)$.

Correlation between the MISS and PSI ratings. The correlation between the actual severity of an injury (MISS) and the potential severity of an injury (PSI) was obtained on the sample of injuries in which both scores were obtained ( $n=$ 2,483 ). Because the number of injuries varied from family to family, observations were weighted by calculating the reciprocal of the number of injuries for each family receiving a PSI score. Results showed a moderate correlation $(r=.39, p$ $<001$ ) between actual injury severity and potential severity (see Figure 2). Further examination of the data indicated that for 1,776 of the injuries, the PSI score was higher than the MISS score. Therefore, in $72 \%$ of the cases, the injury was deemed to have had the potential to be more severe than it actually was. This further supports the notion that minor injuries should not be overlooked, because even in similar circumstances, there is the possibility that the injury could be more

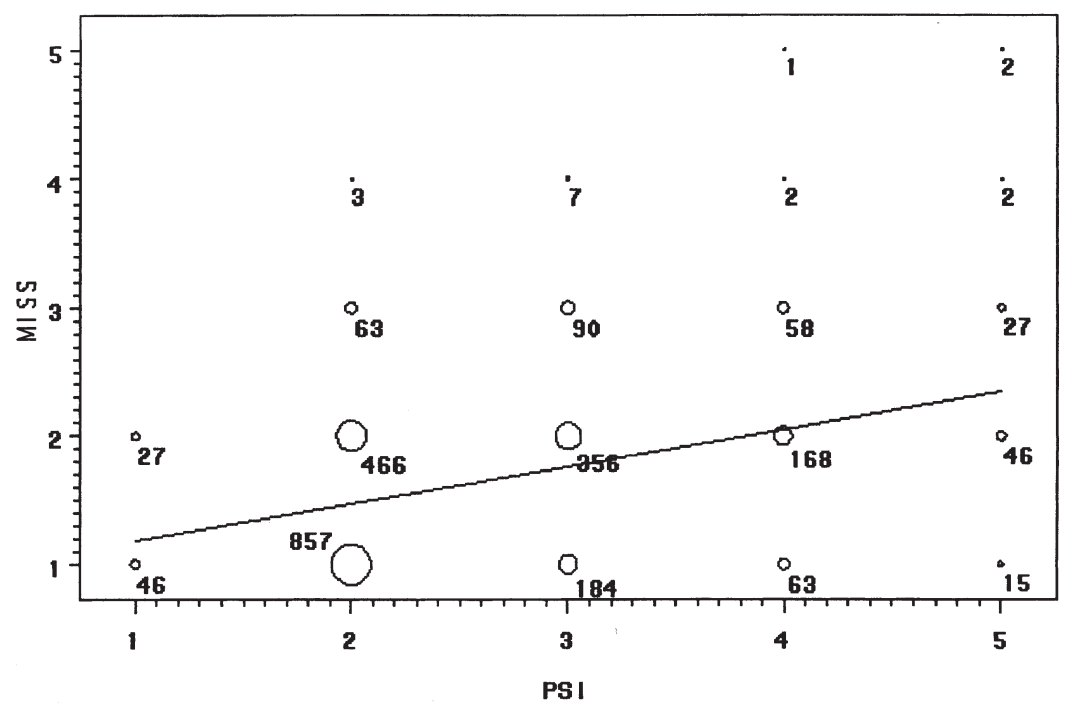

FIG. 2. Comparative distributions of MISS and PSI scores.

severe. As noted above, 92 (nearly 4\% ) of the injuries in this study were rated as having the potential to be fatal or permanently disabling.

Medically attended injuries. Although the majority of injuries in the current study were fairly minor, the PEM does not limit the inclusion of more serious injuries. Twenty-two families reported that the target child had experienced an injury requiring a medical visit or hospitalization during the course of the study (one family reported two medically attended injuries). The severity of these injuries on the MISS $(M=2.82 ; S D=1.22 ; \mathrm{CI}: 2.28-3.59)$ and the PSI $(M=3.27 ; S D=$ 1.35; CI: 2.67-3.87) were larger than injuries that did not receive medical treatment (MISS: $M=1.63 ; S D=.67$; CI: $1.60-1.66$; PSI: $M=2.57 ; S D=.87$; ; 2.53-2.60). ${ }^{3}$ This lends some initial support to the validity of the MISS and PSI. Further, if the mean MISS ratings for medically attended injuries serve as a proxy that may indicate the need for medical attention $(M=2.8)$, then 258 nonmedically attended injuries met similar criteria, in terms of exceeding the 2.8 mean by scoring a 3.0 on the MISS. Similarly, 9 of the 22. medically attended injuries received MISS scores that indicated very little tissue damage had occurred (i.e., MISS ratings less than 3 ). This further illustrates that tissue damage may not be the only factor considered when parents are subjectively considering when the need for medical attention is necessary in response to an injury.

${ }^{3}$ It is inappropriate to conduct $t$ tests in this case due to the confounding of between- and within-subject observations and the lack of overlapping confidence intervals. 
Interventions. A central purpose and advantage of PEM is its ability to assess how often parents actually intervene to make the environment or child safer and to measure the specific efforts parents take to prevent childhood injuries. To examine these issues, participants were asked to report on whether they had intervened (longer than 30 seconds) with the child or the environment either following a specific injury (reactive) or before an injury could occur (proactive). Despite reporting nearly 4,300 injuries, parents reported only 238 reactive interventions (interventions made after an injury occurred to prevent re-injury). Of these reactive interventions, $24 \%$ were reactive barriers, $27 \%$ were reactive alterations of maternal habits, and $48 \%$ were reactive teaching behaviors. Note that the low number of reactive interventions suggests that parents take active efforts to prevent re-injury less than $6 \%$ of the time. It is important to recall, however, that the definition of a reactive intervention is somewhat restricted in that parental efforts must have lasted a minimum of 30 seconds.

Given that there were relatively few interventions involving more than a few seconds even when an injury might have cued them, it might have been expected that there were a very small number of proactive interventions when no injury provided a cue for the need for increasing child safety. In contrast to this supposition, the 170 participants reported 1,345 proactive interventions, interventions that were implemented to protect the child from an injury that presumably had not occurred. Twenty-eight percent of the proactive interventions were proactive barriers (e.g., baby gates), 21\% were proactive alterations of maternal habits (e.g., increasing supervision in high injury-risk situations), and 50\% were proactive teaching measures (e.g., safety instructions to the child).

To investigate the determinants of proactive interventions, a 2 (child gender) $\times 2$ (child age) $\times 2$ (season of study enrollment) $\times 12$ (interview) repeated-measures ANOVA was conducted (see Figure 3). Proactive interventions tended to decrease over the course of the study, $F(11,1672)=8.16, p<.05$; no other main effects or interactions were significant. It is possible that this decline is due to the implementation of interventions that require a single adjustment (e.g., installing cabinet locks) and consequently do not require continued effort or implementation. Surprisingly, there were no significant main effects for child age or gender. It appears that at this young age, parents intervene similarly with boys and girls even though boys have a higher injury frequency than girls. None of the 2- or 3 way interactions was significant. Parallel analyses were not conducted for reactive interventions due to the low occurrence rate of these interventions that resulted in zero cell frequencies.

\section{The Association Between Injuries and Interventions}

An important extension of this study from many past behavioral assessments is the examination of the relationship of interventions to injuries. If interventions are not related to injury rates, then perhaps they are not worth the effort, financial cost, etc., associated with preventive efforts.

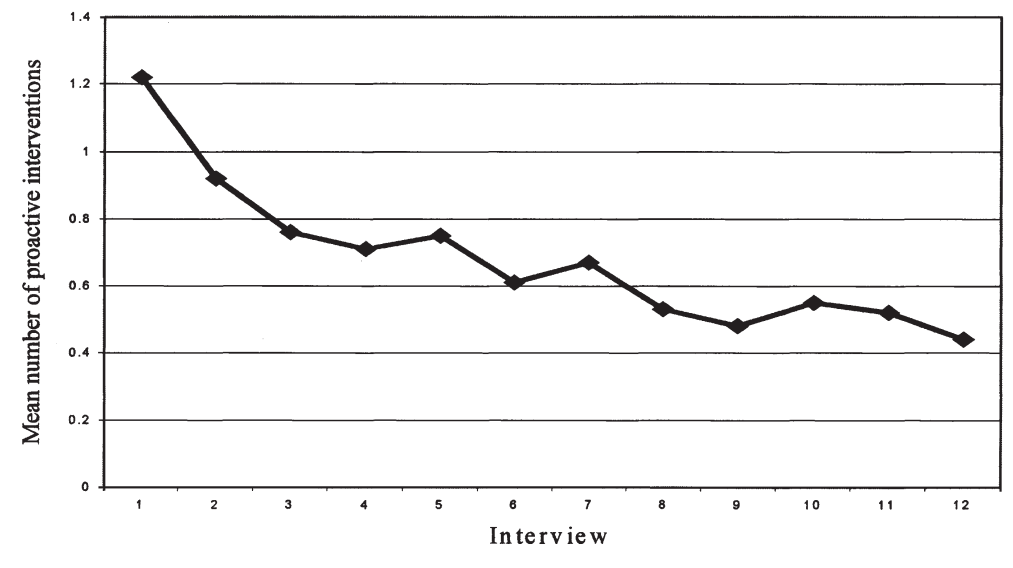

FIG. 3. Mean number of proactive interventions per interview.

Reactive interventions. We computed a correlation in order to assess the relation between reactive interventions and injuries. Results showed that reactive interventions were positively associated with injuries $(r=.26, p<.001)$. This pattern was consistent for specific types of interventions as well. That is, the use of reactive barriers was associated with more, not fewer, injuries $(r=.16 ; p<.05)$, as was the use of reactive teaching $(r=.18 ; p<.05)$ and reactive alterations of maternal behaviors $(r=.21 ; p<.01)$. Again, it should be noted that by definition an intervention was classified as reactive only if an injury had occurred. Thus, there is an inherent dependence of these two variables and results should be interpreted with this in mind. The next section examines interventions that are one step removed from an explicit injury, this propelled by the same motive as reactive interventions, how to prevent injuries in the future.

Proactive interventions. Correlational analyses were conducted as a preliminary step in determining if injuries decreased as proactive interventions increased and whether the implementation of interventions was facilitated by previous occurrences of injury. Contrary to expectations, results revealed a low but positive association between proactive interventions and injuries $(r=.17, p<.05)$, indicating interventions were related to increased rates of injury. Similar patterns were noted when considering specific types of proactive interventions. For example, the use of proactive barriers showed a low but positive association with injuries $(r=.16$, $p<.05)$, as did the use of proactive teachings $(r=.19, p<.05)$. However, alterations of maternal behaviors were not significantly associated with injuries. These findings are somewhat surprising in the sense that one would expect proactive interventions to be associated with a decrease in injury rate. Examination of mothers' reports of where they got the idea for proactive interventions indicated that in $26 \%$ of the cases, the mother was responding to a situation in which an injury almost occurred (see Table 3 ). Thus, the positive association of injuries and proac- 
tive interventions may suggest that mothers intervene frequently with some children because those children are at high risk for injury or have children who often put themselves in dangerous situations in which they "almost" get hurt. Therefore, it may be difficult to distinguish those interventions that are truly proactive in the sense that an injury has not occurred from those that may be conducted in response to a near-injury or prevention of an injury that is about to occur. The data also suggest that given that neither reactive nor proactive interventions resulted in reduced injury rates, mothers' methods of intervention may be relatively ineffective.

\section{Project Feedback Data}

The post-study questionnaire was completed by 120 of the original 170 mothers (others could not be reached or declined to come in to the research laboratory where the questionnaires could be completed and turned in in a fashion guaranteeing their anonymity). The questionnaires were completed 5 months to 19 months after the end of participation. They yielded extensive information suggesting that participants had indeed completed the study as requested. Participants indicated that they kept accurate records regarding injuries close to the event $(M=2.33, S D$ $=1.26$, on a scale where $0=$ recorded every incident as soon as it occurred, $3=$ almost always recorded incidents within a day, $6=$ sometimes recorded incidents within a few days, and $10=$ rarely recorded incidents). Their rating for interventions on the same scale was only a little more lenient $(M=3.48, S D=2.10)$. Parents indicated they gave injuries and interventions careful thought, that they did not deliberately fail to report injuries or interventions, and did not report events that did not occur. They tended to believe that the project had not made them either more aware of or worried about safety issues $(M=6.86, S D=1.43$ for aware $M=5.92, S D=1.24$ for worried) on a $1=$ less, $5=$ no change, to $10=$ more scale. The large majority $(91.8 \%$ ) said they would participate in the study again if asked to do so.

In summary, PEM allowed mothers to be active participants in the data collection of over 4,000 injuries. Although the actual tissue damage resulting from these injuries was fairly minor, approximately $72 \%$ of the injuries had the potential to be more severe under identical circumstances, and 92 (4\%) of the injuries measured in only a 6-month period had the potential to be permanently disabling or even fatal. Mothers reported a surprisingly low number of interventions to prevent re-injury but reported having implemented over 1,300 proactive measures to prevent a first-time occurrence of an injury. When asked anonymously, most mothers reported using the PEM method accurately and rated the experience positively (e.g., they would do the study again).

\section{Discussion}

The extension of the PEM method allows the researcher to (a) focus on injuries in very young children, who are at most risk for serious injuries (Rivara,
1982) and death (Baker et al., 1992); (b) consider two goals of prevention, reactive and proactive, and their methods of achieving prevention through environmental change, maternal behavioral change, and alterations in supervision; and (c) compare settings in which injury occurs, to settings on another (control) day, in which no injury occurs. These goals were selected as the most important to identifying behavioral changes in caregivers that can safeguard children in the future.

Behavioral interventions to improve parenting rely on detailed, objective, and specific aspects of data. Consider the data collection measures that have been reviewed in this paper and their ability to provide such data. Epidemiological methods produce a very large number of participants, but because this method is so diffuse, it cannot provide depth of information regarding specific mechanisms of injury necessary to plan behavioral interventions. Using trained observers who follow up on incomplete answers would be expected to be more accurate than medical records written for purposes other than research (e.g., physical follow-up, insurance reimbursement). It seems especially important to have a nonsubjective measure of the seriousness of the injury, not possible in all but the most serious (top .02\%) of these kinds of cases where a child is disabled or killed. Although severity in such cases is clear, there are too few injuries to analyze specific details to plan behavioral interventions. Even though examining medically attended injuries rather than disabling or fatal injuries would have increased the numbers somewhat, there still would have been too few injuries to study. In our study, there were 10 times as many injuries at the typical level of medically attended injuries (the mean severity of medically attended injuries was nearly 3 , where injuries in the study ranged from 1 to 5) than there were medically attended injuries and injuries at the same level of severity not medically attended. Thus, the PEM allows measurement of the factors surrounding injuries at all levels of severity, where severity is measured objectively.

Examples of the Relationship of Number of Injuries to Past Major Variables Such as Gender

The PEM replicated the findings of several past studies; for example, it confirmed a difference in gender wherein boys were injured more often than were girls. This has been one of the findings most often reported for major injuries in epidemiological studies (Rivara, Calonge, \& Thompson, 1989). No age differences were predicted for number of injuries in these age groups. Rather, they were selected because some research (e.g., Gralinski \& Kopp, 1993) suggests that parents intervene more often in younger than older preschool children. However, we were unable to detect age differences in this sample. This is consistent with epidemiological studies that typically combine toddler (ages 1 to 3 ) and preschool (ages 3 to 4) ages (Baker et al., 1992; Baker \& Waller, 1989). These children are likely to be similarly at risk, in that they share such characteristics as impulsivity, inability to recall rules, and lack of parental verbal control over child behavior. 


\section{Characteristics of Injuries}

Nearly three quarters of the injuries reported were bumps and bruises or cuts and scrapes, and the others fell into the other 16 types of injuries measured by the MISS. Similarly, the large majority (over 94\%) were fairly minor injuries, with few scored as fairly severe. The finding that medically attended injuries were more severe lends further validity to the severity ratings. However, nearly a third of the injuries were rated as having the potential to be serious-many more than would be picked up by any other sampling method. There was a moderate correlation between actual and potential severity, as was expected. Severity as measured by the MISS is an index of damage actually done. For planning prevention programs, severity and even more so, potential severity may have more important implications.

\section{Characteristics of Interventions}

Given that 14 million children a year are hospitalized, some permanently disabled or killed by injuries, the central purpose in examining injuries is to learn about interventions to prevent these unfortunate events. Few studies in the past have examined parents' preventive interventions at all, even by self-report. Other than the studies looking at permanent barriers (e.g., fences around swimming pools), there are only a few studies that have observed interventions focused on brief responses in which parents may take an object away from a child or give a short verbal reminder, (e.g., "We don't touch knives, they are sharp"), either method taking only a few seconds and neither of which seems likely to have a lasting effect on children's injury risk. When focusing on type of brief (lasting only a few seconds) interventions, the research studies considered earlier suggested that mothers responded to children to avoid future risk and that they did so frequently.

Effortful interventions. When mothers in the present sample were asked about more effortful interventions following an injury (i.e., those lasting over $30 \mathrm{sec}-$ onds), they reported a relatively small number, just over 200 reactive interventions or interventions that occurred as a specific response to any of the 4,000 injuries reported. Although it may be surprising that injury events elicit parental interventions for only about 1 per every 20 injuries, some conventional wisdom suggests reasons for the low rate of reactive preventive interventions. For example, parents may believe that once an injury occurs, it may be that the injury itself is preventive (e.g., recall Benjamin Franklin's aphorism "Once burned, twice shy") Some of our past work suggests a relationship between parents' belief about the role injury plays in avoiding further risk and the number of injury prevention rules (Peterson \& Schick, 1993).

In contrast, six times as many interventions were reported to be proactive rather than reactive. That is, over 1,300 interventions were evaluated not to be directly related to a specific injury that occurred; rather, mothers used them to avoid the possibility of an unspecified future injury. Although we asked the mothers to re- cord where the concept for prevention came from and asked about the source of interventions, a quarter of the time the mothers were responding to a "near injury" (Peterson, Brown, et al., 1996), and what Morrongiello (1997) calls "close calls." Close examination of past research considered in our introduction suggests that this may be a closer match to those minimal, preventive observational studies (e.g., Gralinski \& Kopp, 1993) in which the intent is simply to prevent the chance of an injury event and not to alter future behavior. In well over half of the remaining reports, the mother did not cite a specific source of information motivating a proactive intervention, but suggested that her preventive intervention was only common sense or she had thought of the intervention herself. In many of these cases, however, the original source of the intervention (e.g., TV, friend, relative, etc.) may simply not have been recalled by the mother or the effects may have been cumulative (e.g., no single motivation could be cited).

Kinds of interventions. Regardless of whether parents reported an intervention to be reactive or proactive, they were queried about the three potential kinds of interventions described earlier, As was noted in the introduction, interventions fall along a continuum of caregiver effort: Barriers were likely to involve the least ongoing effort and to be the most effective; however, they were used only about quarter of the time, Maternal changes in the mother's own behavior were the next effortful and accounted for another quarter of the interventions. Teaching, probably the most effortful and the least likely to be effective with children of this age, was used half of the time; that is, teaching was used at twice the rate of the other two types of interventions. If parents are going to rely on teaching so often, researchers in the future should ask questions concerning the average mother's understanding of younger children's ability to understand, recall, and respond to verbal commands, and other social variables. Most important, mothers should understand that for children under age 4, a "family rule" discussed weeks or months ago was very unlikely to control even the most compliant child, as children of this age are not cognitively capable of words (especially those spoken some time ago) controlling their behavior.

Findings indicate a decline in interventions, similar to that reported for the number of injuries reported over the course of this study, This may indicate parents' beliefs that their implementation of barriers or other proactive methods result in a safer environment. Once parents have put a proactive intervention in place, they may consider that sufficient protection and fail to follow-up further, However, literature suggests that with children's increasing curiosity and mobility, parents should engage in ongoing efforts to keep their child safe.

\section{Limitations}

Although this study has many advantages as a novel method of collecting data regarding injury and intervention prevalence and interactions, it clearly has common limitations. The sample is made up of primarily middle-class, Caucasian, and 
relatively well-educated parents from a small midwestern city. Despite considerable effort, we were unable to get participation from a greater number of low-income mothers and minority mothers. It is possible that such mothers did not have a telephone required for screening, did not come in contact with our advertisements or requests for participation in parent groups, or may have been ineligible because they had more children (and thus were less likely to have only one child within the 10-year range). Low income parents may also be more wary of authority figures evaluating their parenting practices, as they have witnessed others in their community having children removed from their homes due to neglect (Peterson et al., 1997). Further, considerable effort was involved in the present study, which at times almost became like a second job. For a lower SES household where organization and energy are low, this may have been more difficult. Interestingly (perhaps because of the sound clinical nature of the relationships established by the interviewers), only 9 of the 170 dropped out of the study once they began. Future research may want to focus on recruiting such families specifically because they may be more at risk and are underexplored.

A second limitation is the lack of inclusion of fathers. Although fathers are increasingly more active in the rearing of their children, mothers remain the primary caregiver and spend more time with the child (Straus, 1994). However, future research could be enriched by including fathers as well.

The pattern of fewer injuries being reported across sections was much larger seen in our group beginning in the spring/summer months (we might expect that as children stop playing outside, fewer injuries occur). We must consider that even the small shift seen in our group that began during the fall may have been due either to parents becoming tired of reporting injuries or becoming more reactive to social desirability of reporting fewer injuries, although in an anonymous debriefing parents denied failing, because of boredom or social responsibility, to report injuries or interventions across time. It might even be expected that as the strength of the clinical relationships grew across months, social desirability would have less of an effect, if it had one at all. This remains for future research to determine. If the change was in parents' reports and not due to an actual decrease, they may have been unaware of their own shift in defining injury and intervention. Also, the only change we found in our year-long initial study with 8-year-olds was an increase, not a decrease, in the third season, spring to summer (Peterson, Brown, et al., 1996). It may have been, given that younger children are more at risk for injury, that the injury was due to simple maturation (there is not a large developmental shift from 8 to 85 years, but there is from 18 months to 2 years old).

Finally, the data are a direct product of maternal self-monitoring. These records may be less subject to typical mistakes in self-report due to factors such as faulty memory, because the PEM uses (a) extensive training, (b) the records made at the time of the injury, and (c) biweekly collection of the data. Distortions in recall can still occur, however, and there may be a press to give socially appropriate answers (although mothers denied this in an anonymous follow-up questionnaire).
It remains for additional study to determine how these and other potential limitations can be focused upon and even stronger and more specific data retrieved.

\section{Future Research}

The primary intent of this article was to demonstrate the breadth and depth of the PEM, including proximal (current, state) and distal (temperament, trait) measures of data collection. Only a few of the major variables that were collected were considered here as examples of the richness of these data. It remains for future research to assess whether there are larger numbers of minor as well as more severe injuries in children as they grow up, to determine whether the type of injuries change, and to examine whether gender effects remain the same across children of different ages. The relationship of actual and potential severity of injury needs to be continually examined.

Additional research can examine other data from this method of collection. The impact of different caregivers within different childhood activities offering differing levels of supervision, etc, are all proximal (specific to the situation) factors that could yield valuable information involving the potential for injury. Similarly, more distal or trait-like factors such as child temperament, maternal psychopathology, etc., can contribute to the ultimate judgment of injury risk. One of the advantages of the PEM is that there is relatively little participant burden; the participant need only write brief notes to allow the trained interviewer to glean a variety of information regarding types of interventions, including longhand, stream-of-conscious data, to be coded into more complex data.

PEM ultimately yields clues about effective methods of preventing all levels of severity of injuries in the future. Currently, there are no data to prove or disprove the contention that knowledge of the antecedents, events, and consequences of a minor injury differ from a major injury, except for the tissue damage inflicted as a consequence. Our ratings of injury potential support a premise of no difference. One of the initial important discriminations will be ascertaining the levels at which information gleaned from any given injury event can result in injury preventive information in the future. But the ultimate test will be to determine the extent to which factors identified by PEM can be linked to more effective childhood injury programs, which can identify the systems that can then be used to document the differential influence of various preventive interventions in the future.

\section{References}

Baker. S. P., O’Neill, B., Ginsburg, M. J., \& Li, G. (1992). The injury fact book. Lexington, MA: Lexington Books.

Baker, S. P., \& Waller, A. E. (1989). Childhood injury: State by state mortality facts. Baltimore: The Johns Hopkins University School of Public Health.

Canadian Children's Safety Network. (1996). Parental attitudes toward unintentional childhood injuries. Thousand Oaks, CA: Sage Research Corporation (H11931aB). 
Christoffel, K. K., Scheidt, P. C., Agran, P. F., Kraus, F. J., McLoughlin, E., \& Paulson, J. A. (1992). Standard definitions for childhood injury research: Excerpts of a conference report. Pediatrics, 89, 1027-1034.

Consumer Product Safety Commission. (1979). Impact of crib safety activities on injuries and deaths associated with cribs. Washington, DC: Author.

Deal, L. W., Gomby, D. S., Zippiroli, L., \& Behrman, R. E. (2000). Unintentional injuries in childhood: Analysis and recommendations. In R. E. Behrman (Ed.), The future of children (pp. 4-22). Los Angeles: David and Lucile Packard Foundation.

Eichelberger, M. R., Gotschall, C. S., Feely, H. B., Harstad, P., \& Bowman, L. M. (1990). Parental attitudes and knowledge of child safety. American Journal of Diseases in Children, 144, 714-720.

Finney, J. W., Christophersen, E. R., Friman, P. C., Kalnins, I. V., Maddux, J. E., Peterson, L., Roberts, M. C., \& Wolraich, M. (1993). Society of Pediatric Psychology Task Force report: Pediatric psychology and injury control. Journal of Pediatric Psychology, 18 499-526

Gralinski, J. H., \& Kopp, C. B. (1993). Everyday rules for behavior: Mothers' requests to young children. Developmental Psychology, 29, 573-584.

Louis, T. A., Lavori, P. W., Bailar J. C. III, \& Polansky, M. (1984). Crossover and self-controlled designs in clinical research. New England Journal of Medicine, 310, 24-31.

Maclure, M. (1991). The case-crossover design: A method for studying transient effects on the risk of acute events. American Journal of Epidemiology, 133, 144-153.

Miller, T. R., Romano, E. 0., \& Spicer, R. S. (2000). The cost of childhood unintentional injuries and the value of prevention. In R. E. Behrman (Ed.), The future of children (pp. 137-163). Los Angeles: David and Lucile Packard Foundation.

Morrongiello, B. A. (1997). Children's perspectives on injury and close-call experiences: Sex differences in injury-outcome processes. Journal of Pediatric Psychology, 22, 499512 .

National Institute for Health Care Management. (2000). Preventing childhood injuries for a bright future. Action Brief, August, 1-2.

Peterson, L., Bartelstone, J., Kern, T., \& Gillies, R. (1995). Parents' socialization of children's injury prevention. Child Development, 66, 224-235.

Peterson, L., \& Brown, D. (1994). Integrating child injury and abuse-neglect research: Common histories, etiologies, and solutions. Psychological Bulletin, 116, 293-315.

Peterson, L., Brown, D., Bartelstone, J., \& Kern, T. (1996). Methodological considerations in participant event monitoring of low base-rate events in health psychology: Children's injuries as a model. Health Psychology, 15, 124-130.

Peterson, L., Farmer, J., \& Kashani, J. H. (1990). Parental injury prevention endeavors: A function of health beliefs? Health Psychology, 9, 177-191.

Peterson, L., Gable, S., Doyle, C., \& Ewigman, B. (1997). Beyond parenting skills: Battling barriers and building bonds to prevent child abuse and neglect. Cognitive and Behavioral Practice, 4, 53-74.

Peterson, L., Heiblum, N., \& Saldana, L. (1996). Validation of the Minor Injury Severity Scale: Expert and novice quantification of minor injury. Behavior Therapy, 27, 515-530.
Peterson, L., Moreno, A., \& Harbeck-Weber, C. (1993). "And then it started bleeding": Children's and mothers' perceptions and recollections of daily injury events. Journal of Clinical Child Psychology, 22, 345-354.

Peterson, L., Mori, L., Selby, V., \& Rosen, B. (1988). Community interventions in children's injury prevention: Differing costs and differing benefits. Journal of Community Psychology, 16, 62-73.

Peterson, L., \& Roberts, M. C. (1992). Complacency, misdirection, and effective prevention of children's injuries. American Psychologist, 47, 1040-1044.

Peterson, L., Saldana, L., \& Heiblum, N. (1996). Quantifying tissue damage from childhood injury: The Minor Injury Severity Scale (MISS). Journal of Pediatric Psychology, 21, 251-267.

Peterson, L., \& Schick, B. (1993). Empirically derived injury prevention rules. Journal of Applied Behavior Analysis, 26, 451-460.

Power, T. G., \& Chopieski, M. L. (1986). Childrearing and impulse control in toddlers: A naturalistic investigation. Developmental Psychology, 22, 271-275.

Rivara, F. P. (1982). Epidemiology of childhood injuries. American Journal of Diseases of Children, 136, 399-405.

Rivara, F. P., Calonge, N., \& Thompson, R. S. (1989). Population-based study of unintentional injury incidence and impact during childhood. American Journal of Public Health, 79, 990-994.

Rodriguez, I. G. (1990). Childhood injuries in the United States: A priority issue. American Journal of Diseases of Children, 144, 625-626.

Scheidt, P. C., \& Workshop Participants. (1988). Behavioral research toward prevention of childhood injury. American Journal of Diseases of Children, 142, 612-617.

Shields, I. (1997). Have we become so accustomed to being passive that we've forgotten to be active? Injury Prevention, 3, 243-246.

Straus, M. A. ( 1994 ). Beating the devil out of them: Corporal punishment in American families. San Francisco: Jossey-Bass.

U.S. Advisory Board on Child Abuse and Neglect. (1995). A nation's shame: Fatal child abuse and neglect in the United States. Washington, DC: U.S. Government Printing Office. 